Proceedings of the 46th International School and Conference on the Physics of Semiconductors "Jaszowiec" 2017, Szczyrk

\title{
Sub-Terahertz Emission from Field-Effect Transistors
}

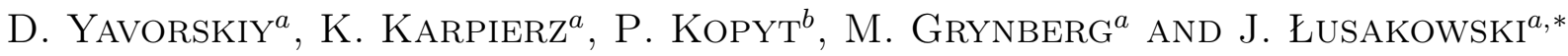 \\ ${ }^{a}$ Faculty of Physics, University of Warsaw, L. Pasteura 5, 02-093 Warsaw, Poland \\ ${ }^{b}$ Institute of Radioelectronics, Technology University of Warsaw, Nowowiejska 15/19, 00-665 Warsaw, Poland
}

\begin{abstract}
Several commercially available field-effect GaInAs-based transistors were studied as emitters of electromagnetic radiation. The emitters were tested either at room or at liquid helium temperature. To spectrally analyse emitted radiation, we applied three different experimental techniques: a spectrum analyser with antennas and mixers, a Michelson interferometer and a magnetic-field-tunable InSb detector. We show that the emission consists of a fundamental frequency of $11.5 \mathrm{GHz}$ and its multiple harmonics spanning the emission band up to about $400 \mathrm{GHz}$. Analysis of the results allows us to suggest that the emission is caused by a Gunn effect and a high harmonics content is related to a pulse-like time dependence of the current.
\end{abstract}

DOI: 10.12693/APhysPolA.132.335

PACS/topics: Field-effect transistors, THz emission

\section{Introduction}

With a rapid development of $\mathrm{THz}$ technology and its applications during the last two decades [1], a quest for semiconductor-based detectors and emitters has become one of the main directions of THz-related research. Although one has to admit that a time-domain spectroscopy based on a fs excitation and detection of semiconductor antennas has largely fulfilled researchers' and commercial requirements [2], there are still many activities where other types of detectors and sources are needed. On the detection side, the situation seems to be quite good since traditionally used Golay cells, liquid-heliumcooled bolometers, and pyroelectric detectors have been accompanied with multipixel devices based on matrices of microbolometers or field-effect transistors. Current parameters of these devices are sufficient to carry out both refined scientific experiments and technical tests.

On the other hand, the source side suffers from essential deficiencies of handy devices if one counts on cheap semiconductor solutions. In fact, all typically used sources: molecular lasers, backward-wave oscillators, and frequency multipliers are very expensive equipment. Looking for possible solutions, one immediately encounters a long-known and not yet resolved problem of a radiative decay of two-dimensional plasmons which has been for decades considered as a promising source of $\mathrm{THz}$ radiation but never clearly proved to exist, in spite of repeating efforts of different experimental groups [3]. Finding a solution to this problem has been still an appealing motivation for researchers also nowadays. Next, a quarter of century ago a new hope for construction of a cheap semiconductor $\mathrm{THz}$ source was awoken by a theoretical prediction of a current instability in nm fieldeffect transistors (FETs) [4]. The beauty of that proposal was that the radiation was predicted to be gate-

*corresponding author; e-mail: Jerzy.Lusakowski@fuw.edu.pl voltage-tunable and spectrally narrow. In fact, following this idea, an emission from nm field-effect transistors was experimentally observed and characterized [58]. Surprisingly, this observation did not lead, however, to construction of a FET-based THz sources, quite opposite to FET-based THz detectors [9]. The reason could be the fact that the emission was weak, hardly tunable with the gate or drain voltage and observed mostly at liquid helium temperatures.

The present paper gives a short summary of a variety of experiments which were carried out at our laboratory in order to observe and analyse a radiation generated by commercially available FETs.

\section{Experimental}

The FETs studied were bought on the free market. According to information given by the producer, the FETs are based on a GaInAs quantum well, the gate length is equal to about $100 \mathrm{~nm}$. A transfer characteristics shows the threshold voltage at about $0.5 \mathrm{~V}$. The FETs were edge-bonded with $\mathrm{Al}$ wires to a home-made multipin support, mimicking a DIL14 standard. The FETs were tested either at $300 \mathrm{~K}$ or $4.2 \mathrm{~K}$. The emission was observed at high drain voltages, in the saturation of the output characteristics. The emission spectrum was analysed with three different experimental systems: a homemade Michelson interferometer, a high-frequency spectrum analyser and a magnetic-field-tunable InSb detector. A beam-splitter in the Michelson interferometer was a 2 inch wafer of a semi-insulating GaAs. One of the mirrors of the interferometer was placed on a linear scanner which could move through the distance of $15 \mathrm{~cm}$ with the step of $10 \mu \mathrm{m}$. We used either a Golay cell or a liquid-helium cooled bolometer as a detector. For tests at $300 \mathrm{~K}$, the FETs were placed directly at the entrance port of the interferometer. Using the interferometer, we applied low-frequency voltage pulses (typically, $7-17 \mathrm{~Hz}$, $50 \%$ duty cycle) to the FET's drain and used a lock-in technique to measure the detector's signal. 
We applied two different experimental techniques for low-temperature measurements when the FETs were placed in a liquid-helium cryostat supplied with a superconducting coil. In one of them, the emitted radiation was guided out of the cryostat through a stainless steel tube which ended at the entrance port of the interferometer. In the other, an InSb detector was placed in the center of the coil and the FET was placed about $30 \mathrm{~cm}$ apart; both the emitter and detector were closed in the same stainless steel tube and the sensitivity of the detector was tuned with the magnetic field [6]. The emitter and detector were cooled with an exchange helium gas or a liquid helium.

Although application of the Michelson interferometer led to convincing and repeatable results, duration of a scan, which in the extreme cases lasted $15 \mathrm{~h}$, precluded registering long series of spectra, for instance, as a function of biasing voltages or temperature. To solve this problem, in the case of room temperature experiments, we coupled FETs to a spectrum analyser working in a few frequency bands up to $220 \mathrm{GHz}$. The coupling was achieved with horn antennas or mixers, chosen appropriately to a particular band. In this case, FETs were biased with a DC drain voltage.

\section{Results and discussion}

One of the major problems encountered in this type of experiments is expressed by the question: does one observe a thermal radiation originating from a Joule heating of the emitter or a radiation caused by an instability of the current flow in the emitter. Generally, there are some signatures of the thermal emission. First, the detected signal should be proportional to the electrical power pumping the emitter. It means that the thermal emission signal should not appear in the threshold manner, when a critical pumping power has been overpassed. In our experiments, we have clearly observed a threshold drain voltage for the emission (about $0.1 \mathrm{~V}$ ). Second, thermal emission cannot be turned on/off too quickly because of a time necessary to cool down the sample to the initial temperature after the voltage pulse had gone. Thus, increasing the frequency of the pulses one should observe a decrease of the emission signal. We could not apply this test because the detectors which we were using are very slow (both the Golay cell and bolometer) while the spectrum analyser required a DC bias of the FET and the InSb detector showed to be useless in our experiments, as it is explained below. Thus, we take observation of the threshold emission voltage as a proof that the emission is due to an instability of the current flow in the FET.

Some characteristics of the observed emission are the following.

1. The emission starts at the drain voltage which falls within the saturation range of the output characteristics in a region of a negative differential con- ductivity observed there. Similar features were observed in [6].

2. The emission spectra consist of a fundamental harmonic of about $11.5 \mathrm{GHz}$ and its multiple higher harmonics. The highest frequency observed (with the Michelson interferometer) was equal to about $400 \mathrm{GHz}$.

3. The transistors generate radiation both at room as well as at liquid helium temperatures, with almost no differences between these two spectra.

4. The spectra of emission weakly depend on the drain and gate voltage.

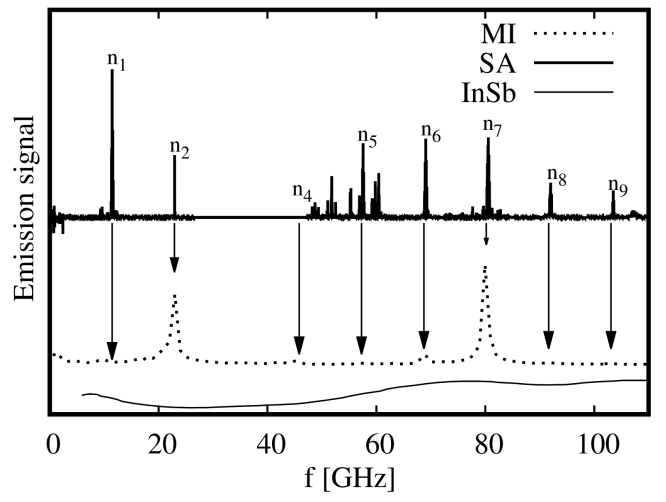

Fig. 1. Emission spectrum registered with three different methods: the Michelson interferometer (MI), the spectrum analyser (SA) and the InSb detector (InSb). The FET was kept at room temperature. $n_{i}$ counts the harmonics of the fundamental frequency.

In Fig. 1 we compare data registered with all three experimental techniques. One can see that the results obtained with the Michelson interferometer and the spectrum analyser are mutually consistent, while they do not agree with the results obtained with the InSb detector. We think that differences in the spectra measured with the interferometer and the analyser result from a much higher sensitivity of the analyser and a totally different electromagnetic coupling of the radiation with the measuring instruments. However, at the moment we cannot present convincing arguments to explain these differences.

An InSb detector has been typically considered as a resonant detector with its response determined by the cyclotron resonance frequency. In other words, one should expect that such a detector responds to radiation of a frequency $f$ only at a magnetic field $B_{f}$ which satisfies the condition $f=e B_{f} / 2 \pi m$, where $e$ and $m$ is the electron charge and effective mass, respectively; for $\mathrm{InSb}$, one finds $f[\mathrm{THz}]=2 B_{f}[\mathrm{~T}]$. This allows to present data measured during a scan of detector's magnetic field as a function of frequency, as it is shown in Fig. 1. Since the spectrum of emission is known, we conclude that a 
response of the InSb detector carries both resonant and nonresonant contributions and such a detector cannot be used for a spectral characterization of low-frequency sources. A more detailed characterization of the InSb detector can be found elsewhere [10].

Taking into account experimental facts and a theoretical analysis found in the literature we propose that the mechanism responsible for the observed emission is the Gunn effect with a high harmonic content resulting from a pulse-like time dependence of the current. We expect that a high electric field necessary to trigger the Gunn oscillations develops between the gate and the drain, as it is in all HEMTs driven into saturation. This distance is of the order of $1 \mu \mathrm{m}$ which allows to estimate the electric field at the threshold of emission for $0.1 \mathrm{~V} / 1 \mu \mathrm{m}=10^{5} \mathrm{~V} / \mathrm{m}$. Monte Carlo simulations of the electron transport in GaInAs heterostructures [11, 12] show that the maximum of the electron drift velocity electric field dependence occurs at the electric field of about $4 \times 10^{5} \mathrm{~V} / \mathrm{m}$ and is accompanied with the onset of population of subsidiary minima in the conduction band, which is a necessary condition for the Gunn oscillations. A saturation velocity is calculated to be equal to about $10^{5} \mathrm{~m} / \mathrm{s}$ which gives the estimation of the frequency of oscillations of $100 \mathrm{GHz}$ which is an order of magnitude higher than observed. However, one has to take into account an extremely approximative character of this estimation and the fact that both numerical $[11,12]$ and experimental observations [13] indicate a strong dependence of the Gunn frequency on material parameters of a particular GaInAs heterostructure considered.

It is true that we cannot supply definite arguments supporting the presented hypothesis. The Gunn effect is a time-of-flight phenomenon and the best proof would be to observe a dependence of the fundamental frequency on the distance through which a Gunn domain moves. This is unfortunately not achievable in the case of commercial transistors studied. A possible experimental verification of a Gunn domain movement over a micrometer distances could be realized by perturbation of its motion with a tip of an AFM. Such a perturbation should lead to changes in the time dependence of the pulsed current and be reflected in a harmonic content of the spectra. An idea of such an experiment comes from an experimental observation of high-electric-field domain movement in semiinsulating GaAs perturbed by a spot of a visible light illuminating locally the investigated sample [14].

\section{Conclusions}

We observed and analysed an electromagnetic radiation from a set of commercially available nanometer
FETs. The emission spectrum consisted of a fundamental harmonic at about $11.5 \mathrm{GHz}$ and many other harmonics with the frequency up to $400 \mathrm{GHz}$. We propose the Gunn effect as a possible mechanism responsible for the observed radiation and suggest a microscopic-type experiment to prove this concept.

\section{Acknowledgments}

A financial support from a Polish National Science Centre UMO-2015/17/B/ST7/03630 grant is acknowledged.

\section{References}

[1] P.U. Jepsen, D.G. Cook, M. Koch, Laser Photon. Rev. 5, 124 (2011).

[2] W. Withayachumnankul, M. Naftaly, J. Infrared Millim. Terahertz Waves 35, 610 (2014).

[3] D.C. Tsui, E. Gornik, R.A. Logan, Solid State Commun. 35, 875 (1980).

[4] M. Dyakonov, M. Shur, Phys. Rev. Lett. 71, 2465 (1993).

[5] Y. Deng, M.S. Shur, R. Gaska, G.S. Simin, M. Asif Khan,V. Ryzhii, Appl. Phys. Lett. 84, 70 (2004).

[6] W. Knap, J. Łusakowski, T. Parenty, S. Bollaert, A. Cappy, V.V. Popov, M.S. Shur, Appl. Phys. Lett. 84, 2331 (2004).

[7] J. Łusakowski, W. Knap, N. Dyakonova, L. Varani, J. Mateos, T. Gonzalez, Y. Roelens, S. Bollaert,A. Cappy, J. Appl. Phys. 97, 064307 (2005).

[8] N. Dyakonova, A. El Fatimy, J. Łusakowski, W. Knap, M.I. Dyakonov, M.-A. Poisson, E. Morvan, S. Bollaert, A. Shchepetov, Y. Roelens, Ch. Gaquiere, D. Theron,A. Cappy, J. Appl. Phys. 97, 114313 (2005).

[9] P. Kopyt, P. Zagrajek, J. Marczewski, K. Kucharski, B. Salski, J. Łusakowski, W. Knap,W.K. Gwarek, Microelectron. J. 45, 1168 (2014).

[10] D. Yavorskiy, K. Karpierz, M. Grynberg, J. Łusakowski, Acta Phys. Pol. A 132, 338 (2017).

[11] J.L. Thobel, L. Baudry, A. Cappy, P. Bourel,R. Fauquembergue, Appl. Phys. Lett. 56, 346 (1990).

[12] J.L. Thobel, L. Baudry, P. Bourel, F. Dessenne,M. Charef, 0.1063/1.355145J. Appl. Phys. 74, 6274 (1993).

[13] A. Khalid, G.M. Dunn, R.F. Macpherson, S. Thoms, D. Macintyre, C. Li, M.J. Steer, V. Papageorgiou, I.G. Thayne, M. Kuball, C.H. Oxley, M. Montes Bajo, A. Stephen, J. Glover,D.R.S. Cumming, J. Appl. Phys. 115, 114502 (2014).

[14] B. Willing, J.C. Maan, Phys. Rev. B49, 13995 (1994). 